\title{
Assist-as-needed Training Paradigms for Robotic Rehabilitation of Spinal Cord Injuries
}

\author{
Lance L. Cai ${ }^{1}$, Andy J. Fong ${ }^{1}$, Yongqiang Liang ${ }^{2}$ \\ and Joel Burdick ${ }^{2}$ \\ 1. Bioengineering and ${ }^{2}$ Mechanical Engineering \\ California Institute of Technology \\ 1200 E. California Blvd. \\ Pasadena, CA 91125, USA \\ Email: \{lancecai/afong/yliang/jwb\}@robotics.caltech.edu
}

\author{
V. Reggie Edgerton ${ }^{1,2,3}$ \\ Dept. ${ }^{1 .}$ Neurobiology, ${ }^{2}$ Physiological Science \\ and ${ }^{3}$ Brain Research Institute \\ University of California, Los Angeles \\ 621 Charles E. Young Dr. South \\ Los Angeles, CA 90095, USA \\ Email:vre@ucla.edu
}

\begin{abstract}
This paper introduces a new "assist-as-needed" (AAN) training paradigm for rehabilitation of spinal cord injuries via robotic training devices. In the pilot study reported in this paper, nine female adult Swiss-Webster mice were divided into three groups, each experiencing a different robotic training control strategy: a fixed training trajectory (Fixed Group, A), an AAN training method without interlimb coordination (Band Group, B), and an AAN training method with bilateral hindlimb coordination (Window Group, C). Fourteen days after complete transection at the mid-thoracic level, the mice were robotically trained to step in the presence of an acutely administered serotonin agonist, quipazine, for a period of six weeks. The mice that received AAN training (Groups $B$ and $C$ ) show higher levels of recovery than Group A mice, as measured by the number, consistency, and periodicity of steps realized during testing sessions. Group $C$ displays a higher incidence of alternating stepping than Group $B$. These results indicate that this training approach may be more effective than fixed trajectory paradigms in promoting robust post-injury stepping behavior. Furthermore, the constraint of interlimb coordination appears to be an important contribution to successful training.
\end{abstract}

Index Terms-Assist-as-needed, Rehabilitative Robotics, Spinal Cord Injury

\section{INTRODUCTION}

Robotically facilitated rehabilitative training paradigms have recently become more common. Commercially available robotic orthotics such as the Lokomat ${ }^{\mathrm{TM}}$ are already available to facilitate the rehabilitative training of spinal cord injured (SCI) and stroke patients with promising results [1]. Additionally, robotic devices are increasingly used for studies on animal models of spinal cord injuries $[2,3]$. Moreover, a growing body of literature suggests that robotic systems and mechanical linkages can play an important role in post-stroke recovery of arm function [4].

However, little is known about the physiological mechanisms of how locomotor recovery is achieved with this type of rehabilitative training. Additionally the best training procedure has yet to be established.

The long term goals of our work are to:

- develop an understanding of the neurophysiological mechanisms underlying locomotor recovery;
- develop the technology and drug therapies to facilitate rapid and optimal recovery;

- develop the best training procedures that lead to rapid and sustained recovery.

Clearly, robotic devices can play an important role in the recovery process. They can provide cost effective therapy (by augmenting the capabilities of human physical therapists), they can quantitatively measure and monitor the key physiological variables related to recovery (thereby giving quantitative assessment of recovery progress), and they can potentially provide adaptive therapies tailored to each patient. Robotic devices can also help automate and systematize the process of spinal cord injury research.

In this paper we use a robotic training device (Fig. 1) coupled with administration of quipazine (a serotonin agonist) to study locomotor recovery after SCI in mice. A mouse model is attractive due to the many strains of transgenic mice available to study detailed issues in the pharmacology of locomotion.

To date, the algorithms that have been used in robotic locomotion training devices have almost exclusively focused on repeated movements of the limbs through fixed kinematic trajectories. However, fixed, repetitive training is very likely to be suboptimal, as it leads to the phenomena of "learned helplessness" $[5,6]$. That is, the lower spinal cord becomes habituated to the training trajectory. Thus, we believe that fixed trajectory training will eventually cause an extensive level of habituation to sensory inputs such that there is eventually little or no response to the sensory inputs imposed by the robotic training device and, therefore, little or no motor output is generated. As a consequence, the training becomes counter productive, causing the rate of rehabilitation to decrease, and preventing the locomotion control circuits from robustly handling stepping trajectories other than the trained pattern [7].

In this paper, we propose and test the hypothesis that the post-SCI spinal cord will be better able to relearn to step if it is constantly challenged during locomotor training by introducing variability in the training pattern. We implement this variability in the form of an "assist-as-needed" (AAN) training paradigm. Our AAN algorithms provide strong 
feedback guidance when the subject's stepping pattern is far from the nominal pattern. However, only a gentle form of guidance is provided when the animal is close to the desired trajectory, thereby allowing the animal to largely guide its own motions when it is doing well. In this way, a reasonable amount of variability in the stepping trajectory is experienced during training, as variability is inherent in normal locomotion. We hypothesize that this form of guidance leads to better and more robust spinal cord learning and locomotion recovery.

There are many possible variations on the AAN theme. The present pilot study compared the efficacy of two AAN robotic training algorithms versus fixed trajectory robotic training on recovery of locomotory ability in completely spinalized adult mice that were administered quipazine. The results of the study show that mice undergoing AAN robotic training exhibited faster and more pronounced recovery than mice given fixed robotic training. These findings suggest that an improved rehabilitation strategy can be realized by combining an "assist as needed" training paradigm and pharmacological intervention. The exact optimal AAN algorithm still needs to be developed. However, the results presented in this paper show the benefits of the AAN paradigm, and suggest avenues for future refinement.

This paper is structured as follows. Section II briefly reviews background on spinal cord injury, and also prior work on the use of quipazine to facilitate locomotor recovery. Section III describes our experimental methods and apparatus, while Section IV details the fixed and AAN training algorithms. Section V describes the techniques used to evaluate locomotion recovery. Section VI summarizes the experimental results, while Section VII discusses our results.

\section{BACKGROUND}

There are $\sim 250,000$ people in the United States suffering from spinal cord injury (SCI), with approximately 11,000 new cases being diagnosed each year [8]. SCI occurs most commonly in young adult males between the ages of 16 and 30 , is most frequently caused by motor vehicle injuries, sports injuries, and falls, and can generate symptoms as severe as paraplegia and quadriplegia. Unlike victims of other types of severe traumatic injuries, SCI patients can live long lives that are severely diminished.

Our research aims to develop robotic and pharmacological strategies for recovering locomotor function in SCI patients that have lost the ability to stand and to step. Numerous animal models of SCI have demonstrated that, even when the spinal cord is completely severed, subjects can be retrained to step as long as specific locomotion-associated neural centers located in the lower portion of the spinal cord are spared by the injury and remain connected to the muscles that produce stepping movements [9]. Even in the absence of descending inputs from the brain, this distributed network of neurons is capable of generating successful stepping. When appropriately modulated by sensory stimuli and drug treatment, this network can even adapt stepping patterns ap- propriately to respond to perturbations in the physical environment. We hypothesize that strategies for treating SCI must include rehabilitation and retraining of these neural centers.

SCI causes substantial changes in the physiological state of the spinal cord. First, depending on the extent of injury, both electrical (via action potentials) and chemical (via neurotransmitters) transmission of information from the brain to the segments of the spinal cord below the lesion are decreased or eliminated. Second, complete SCI is characterized by a near-complete loss of serotonin, a neurotransmitter, below the injury. Locally this loss can hamper the ability of spinal locomotor circuits to appropriately process non-brain sources of information, e.g. sensory information from the lower limbs. Third, SCI is associated with an upregulation of specific biochemical markers, such as Glutamic acid decarboxylase $67\left(\mathrm{GAD}_{67}\right)$, which are associated with increased levels of inhibition in the spinal cord [10]. By creating a general state of inhibition in the spinal cord, SCI may prevent the locomotor network from processing information that is required to generate stepping.

Over the past ten years, numerous researchers have demonstrated that rehabilitative step training can be used to recover locomotor function in cats [11, 12], rats [2], and mice [13]. One important effect of locomotor training is that it reduces the level of inhibitory markers such as $\mathrm{GAD}_{67}[9$, 14]. It is likely that consistent locomotor training generates many other biochemical and physiological changes in the spinal cord, and these changes are currently under investigation.

In our work, we used quipazine, which is a member of a class of drugs called serotonin agonists. The serotonin system is one of the principal neurotransmitter systems of the body, responsible for regulating a diverse array of behaviors. It has been previously observed that pharmacological treat-

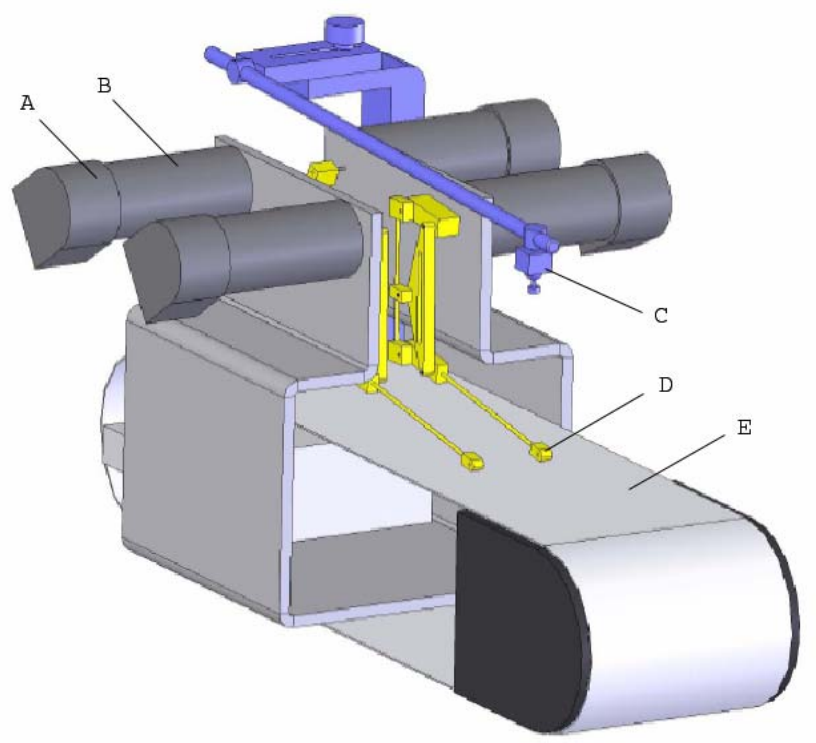

Figure 1: Schematic of current step training system. Important components are labeled: A) Optical encoder; B) Motor; C) Weight support; D) Manipulators; and E) Motorized treadmill. 
ment with the drug quipazine can facilitate stepping in animals whose spinal cords have been completely severed [1517]. More importantly, it has been demonstrated that daily administrated quipazine acutely improved stepping performance, immediately (within ten minutes) enabling mice that were completely unable to step before treatment to execute long bouts of successful, rhythmic, and kinematically appropriate treadmill stepping. Within one week of daily treatment, mice administered quipazine demonstrated statistically significant improvements in number of steps performed, step rhythm, and step shape consistency compared to untreated spinal mice. It is important to note, however, that no stepping movements occurred if the mice were not placed onto a moving treadmill belt. In other words, quipazine did not directly generate the hindlimb movements associated with stepping. Instead, it facilitated sensory processing that is essential for stepping. Additionally, the effects of quipazine were short-lived. The enhanced stepping performance induced by quipazine diminished as the drug was metabolized, decreasing significantly within two hours of treatment, and disappearing by the next day. The observations that quipazine did not directly generate stepping movements, and that quipazine-mediated stepping was only triggered by steppingassociated sensory stimuli such as the movement of a treadmill belt, have led us to hypothesize that quipazine may have an important role in regulating the types and levels of sensory information perceived by locomotion-associated neural centers within the spinal cord. Quipazine treatment may restore appropriate sensory processing, "tuning" the spinal cord to relevant proprioceptive cues, while suppressing extraneous inputs such as pain, thereby enabling stepping to occur.

In addition to its effects in facilitating locomotion, another key function of quipazine is its potential role in spinal learning. The serotonin system is closely intertwined with the N-methyl-D-aspirate (NMDA) system, another important neurotransmitter system that has been implicated for its primary role in memory consolidation and learning [18]. Via its interactions with the NMDA system, quipazine may enable mice that have benefited from robotic locomotor training to retain their stepping ability even long after training has ceased.

It has been observed that providing a combination treatment of robotic training and quipazine treatment yielded better stepping performance than when either treatment was provided alone [13]. Importantly, analysis of these different treatment paradigms demonstrated that the effects of these treatments were distinct, and that they were complementary. These results demonstrate that combining multiple treatment paradigms may be essential to achieving optimal recovery from SCI.

\section{EXPERIMENTAL METHODS AND APPARATUS}

Animals: Our experiment used adult Swiss-Webster mice (mean body weight of $21.5 \pm 1.1 \mathrm{~g}$ at the time of surgery) obtained from Charles River Laboratories (Wilmington, MA)

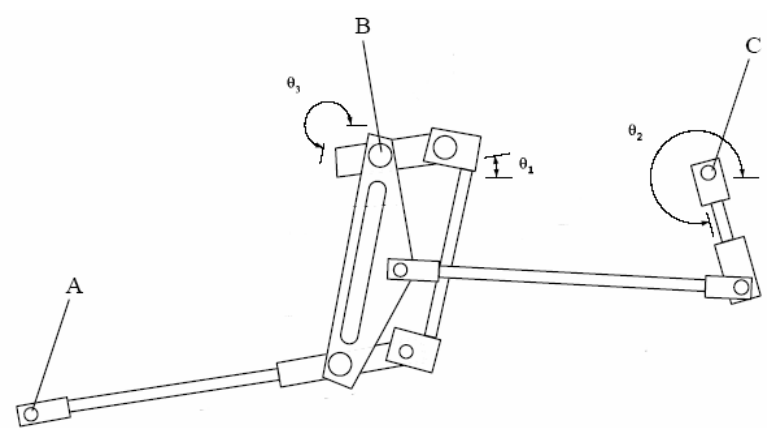

Figure 2: Schematic diagram (drawn to scale) of the 5-bar, parallelogram-type linkage. Labeled features are: A) ankle attachment point; B) motor attachment point driving linkage 4; C) motor attachment point driving linkage 1 .

were used. A complete mid-thoracic spinal transection was performed at 45 days of age.

Quipazine Administration: Quipazine $(0.5 \mu \mathrm{g} / \mathrm{g}$ body weight, i.p.) was administered to all subjects $5 \mathrm{~min}$ prior to each training or testing session [19].

Robotic Step-Training and Measurement System: A four axis robotic system was developed for both active training and data acquisition (see Fig. 1 for a schematic) of mouse limb movements. The robotic system consists of four major components: 1) a pair of 2 DOF robotic manipulators to guide and measure ankle motions in the sagittal plane; 2) motion control hardware; 3) a treadmill; and 4) a body weight-support device.

Each 2 DOF robotic manipulator is comprised of a 5-bar leg guidance linkage (Fig. 2), a pair of motors that drive the linkage, and a pair of optical encoders to record the motors' rotational position. Ankle position is derived from these encoders and the linkage geometry. The five bar limb guidance linkage allows motion sensing and control in a vertical (sagittal) $3.5 \times 3.5 \mathrm{~cm}$ workspace plane, which is sufficient to accommodate all step trajectories associated with mouse locomotion training. In their active mode, the robotic arms drive the hindlimbs. In their passive mode, the linkages move freely in the workspace while attached to the ankles, allowing independent measurements of the ankle movements generated by the mouse.

Due to the linkage geometry, the dynamics of the two axes are approximately uncoupled and approximately linear in the range of motions encountered during training and evaluation. To minimize encumbrance on the limbs of the mice during passive operation, precision bearings and motors with low internal friction were used at all revolute joints. We estimate the frictional resistance force at the end-effector to be $0.032 \mathrm{~N}$. We estimate the mass inertia of the robotic arm linkage, including its actuators, to be $\sim 0.4 \mathrm{~g}$ in its home configuration. Although mass inertia varies with orientation, the maximum mass inertia felt by the mouse is on the same order of magnitude as the home configuration value, and hence is sufficiently small to allow us to assert that the robotic arms 
do not critically hinder stepping.

The mice are placed into the stepper using a coneshaped cloth harness. Once the mouse has entered the harness, it is secured using a binder clip, which is then magnetically attached to the weight-support system. The legs of the mouse were connected to the robotic arm using a drawstring loop attachment.

Experimental Design: Nine mice were randomly divided into three groups. All three groups received a form of robotic training with quipazine for six weeks. The first group was the control in this experiment and was trained with the fixed robotic training algorithm. The two other experimental groups were each trained with a form of AAN training. Each of the spinal mice groups were trained for $10 \mathrm{~min}$. per day, 5 days per week, for a period of 6 weeks. Measurements of stepping performance were carried out on the $6^{\text {th }}$ day of each training week

\section{Robotic Training ALgorithms}

We implemented three different training algorithms, a repetitive training with a fixed and tightly controlled trajectory and two different types of assist-as-needed (AAN) training algorithms. The two AAN algorithms differed in the amount of interlimb coordination that was imposed during training. The AAN training algorithms were implemented using a velocity field approach where the velocity of the linkage's distal tip is commanded to a specific speed defined by a velocity field. Using the linkage Jacobian matrix, the distal velocities are converted to desired motor velocities.

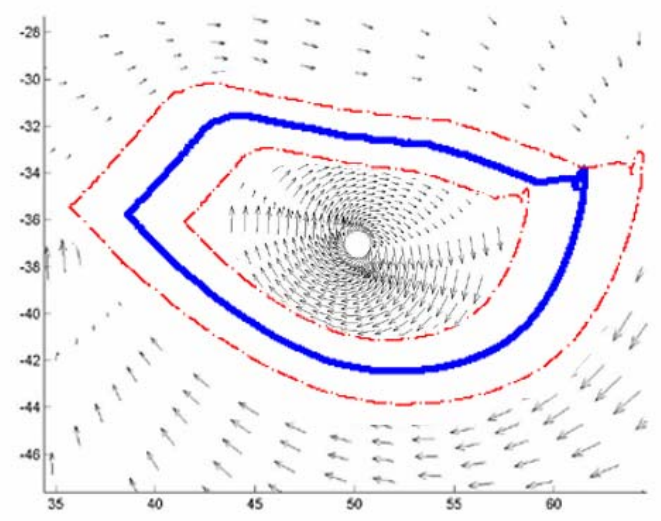

Figure 3: Variability training paradigm I (Band). The desired training trajectory is shown in thick blue. The dotted thin red lines represent the boundaries within which "soft control" is applied to the limbs. The blue arrows correspond to the convergent velocity fields that drive the leg to the band region. (Modified from Cai et al, 2005 [16].)

A. Fixed Robotic Training: In this training method, a PID controller performs continuous tracking of a pre-determined pattern. When attached to the mice, the robotic arms actively move the ankle points of the limbs along this fixed trajectory. The imposed $\mathrm{x}$ and $\mathrm{y}$ trajectory of each hindlimb was obtained from a neonatally transected mouse that stepped well. Neonatally transected mice can spontaneously recover functional stepping without pharmacological or mechanical assistance. This pattern was recorded from the neonatal mouse when it was approximately the same age as the adult mice used in this study.

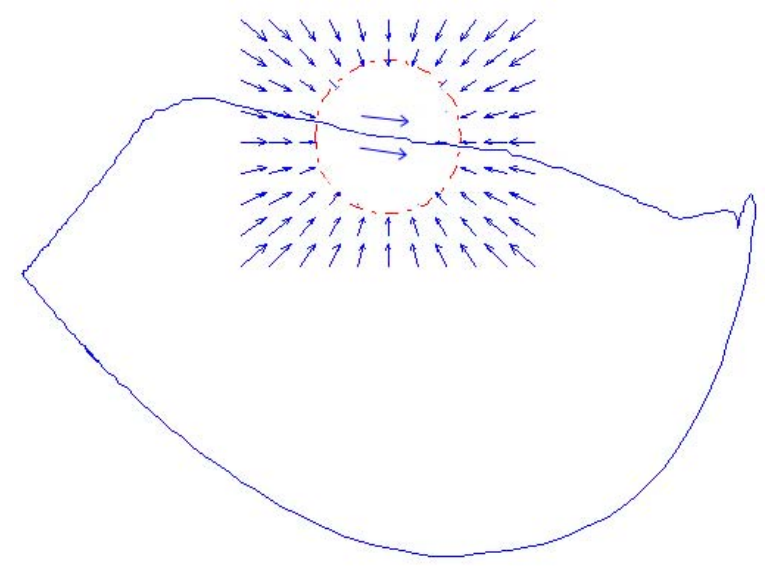

Figure 4: Variability training paradigm II (Window). The desired training trajectory is in blue and the moving window is highlighted in

B. Robotic AAN Training Algorithm I, "Band": This AAN strategy implements two fixed boundaries: an inner bound and an outer bound, forming a "band" surrounding the desired trajectory. When the ankle lies in sagital plane regions inside (outside) the band, an outward spiraling (inwardspiraling) converging velocity field drives the ankle to the band region. When the ankle leaves the band, the convergent velocity fields will rapidly move it back into the band region (Fig. 3.). Within the band, the ankle is guided by a small constant velocity field tangent to the desired trajectory, i.e. the robot nudges the ankle along the trajectory at a constant speed, but does not enforce specific timing of leg movement along the trajectory (Note, the velocity fields inside the band are omitted in Fig. 3 for clarity). In this way, the animal largely dictates its own motions inside the band, with a small bias provided by the robot. Note that this particular instantiation of the AAN paradigm does not impose an interlimb coordination constraint. The computational procedure to construct the velocity field for a given stepping trajectory is outlined in Appendix A.

C. Robotic AAN Training Algorithm II, "Window": The third group received an AAN training paradigm analogous to the second group, but based on a moving "window" geometry. In this approach, a circular "window" moves along the desired trajectory to provide loose timing between the two limbs (Fig. 4). The window size was chosen to be $5 \mathrm{~mm}$ in diameter, and was fixed throughout the experiment. We chose this window size because, from observation, it was close to the typical variation of a normal stepping animal. Within the window, a small constant velocity field tangent to the desired trajectory biases the robot's motion, but does not 
enforce specific timing inside window. Outside the boundary, the robotic movement is guided by a radial force field that points inward with a magnitude proportional to the distance from the circle center: $v=k(d-r)$, where $v$ is the velocity field magnitude, $d$ is the distance between the ankle point and the center of the moving window, $r$ is the window radius, and $k$ is a constant. Hence, when the animal's ankle deviates from the window, it is quickly returned to the window. Within the window, the ankle is gently guided in the direction of the trajectory, thus providing loose timing control. The same strategy was used on both hindlimbs, and the control systems for each leg was timed to provide alternating phasing that is consistent with weight bearing stepping.

\section{DAta AnAlysis AND Evaluation Methods}

We assess the quality of a subject's training in terms of: 1) the number of steps taken by a subject; 2) the periodicity of the subjects steps (i.e., the ability to maintain a regular stepping frequency); and 3) the regularity of the subject's stepping patterns. The following analyses were applied to the data obtained during the weekly tests of each subject (where the robotic trainer is used in its passive mode).

Number of Steps: Video footage and plots of ankle position data were used to identify and count the number of steps performed by each mouse. Steps were identified based upon pre-determined criteria for step length, height, duration, and degree of interlimb coordination. On each testing day, the 12 -sec stepping interval containing the most steps was recorded for subsequent analyses. Both plantar and dorsal steps were accepted. Better subjects performed primarily plantar steps, whereas poorer performing subjects exhibited dorsal steps and paw drag. Immediately after the transection surgery, none of the mice were able to execute any steps. To varying degrees, all of the mice improved their number of steps as a result of training.

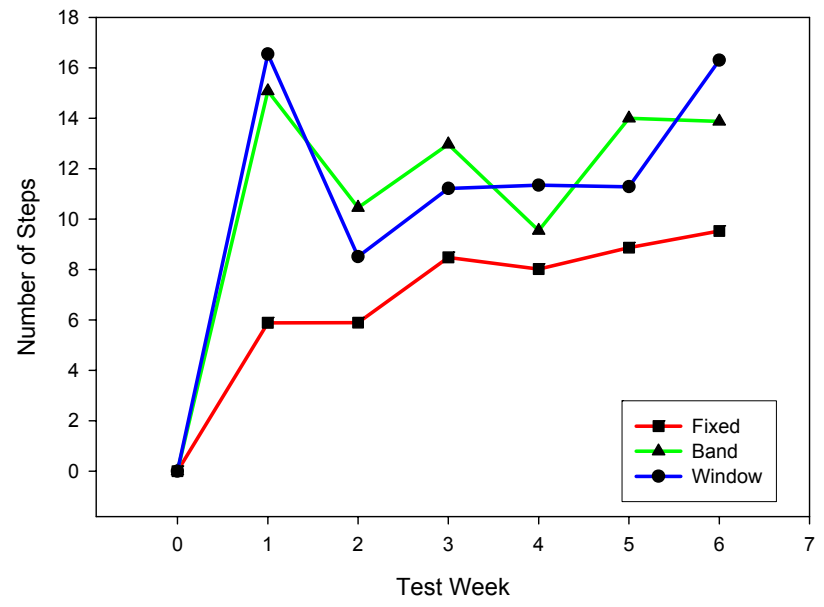

Figure 5: Number of steps performed in $15 \mathrm{sec}$. interval.

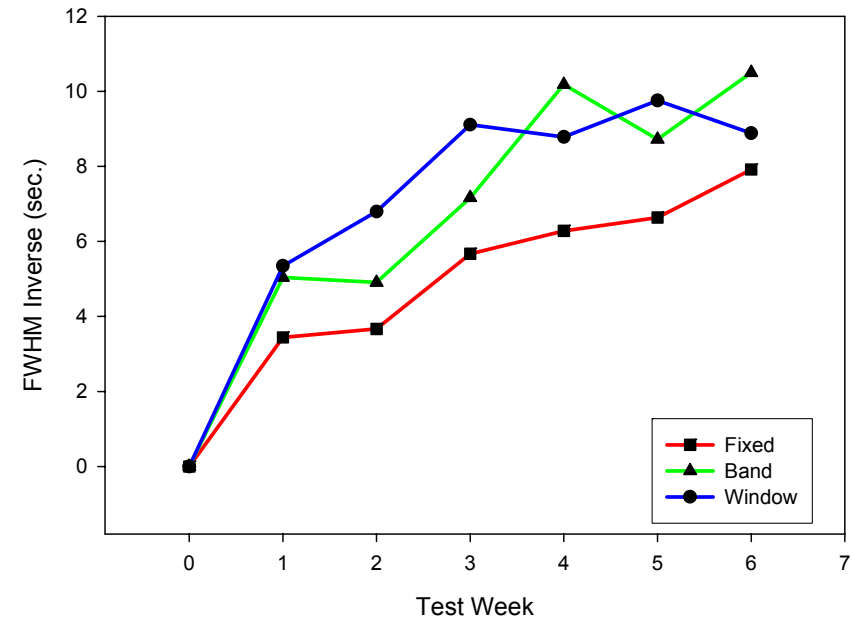

Figure 6: The inverse of FWHM is plotted to emphasize the locomotor improvement over time.

Step Periodicity: We used the Fast Fourier Transform (FFT), applied to the horizontal component of the stepping trajectories, to quantify step periodicity. Mice that stepped rhythmically exhibited a sharp and distinct fundamental peak in the FFT of their ankle trajectories. The location of the predominant peak corresponds to the most common stepping frequency. Conversely, mice with poor periodicity exhibited either a very broad fundamental peak in the FFT spectrum or, in extreme cases, failed to demonstrate a fundamental peak. To quantify these observations, we measured the full width at half maximum (FWHM) of the fundamental peak. A low FWHM value corresponds to temporally consistent, rhythmic stepping, whereas high measurements typically indicate erratic stepping consistent with stumbling and foot dragging.

Spatial Consistency: In addition to the temporal information provided by FFT analysis, we also sought to study changes in spatial stepping consistency throughout the study. We suggest that locomotor improvement can be characterized by more consistent repetition of a nominal trajectory. To measure this quantitatively, we used Principle Components Analysis (PCA). First, we separated the stepping trajectories into their horizontal (or X) and vertical (or Y) components. Next we resampled all the component trajectories so that each test trajectory data set consists of the same number of data points. In this way, only spatial information on the stepping cycles is retained. Then the data are arranged in a matrix form with each columns corresponds to the data points for each $\mathrm{X}$ trajectories of a step and the matrix will have the same number of columns as steps performed with in the ten second interval. For example, given $\mathrm{n}$ steps with $\mathrm{m}$ data points in each, the $\mathrm{X}$-trajectory data matrix matrix will take the following form (with an analogous matrix for the Ytrajectory data). 
$\boldsymbol{X}$ component of the steps

$$
\left[\begin{array}{ccccc}
x_{1,1} & x_{1,2} & \cdots & \cdots & x_{1, n} \\
x_{2,1} & x_{2,2} & & & x_{2, n} \\
\vdots & & \ddots & & \vdots \\
\vdots & & & \ddots & \vdots \\
x_{1, m} & x_{2, m} & \cdots & \cdots & x_{n, m}
\end{array}\right]
$$

We computed the principal components (PC) of the data set, as well as the percentage of the total variance in the data set that is captured by the first principal component. The first principal component can be interpreted as the "nominal" trajectory from the test data set, and the PCA percentage score measures how repeated are the trajectories. A high PCA percentage score corresponds to a subject that consistently executes the same stepping trajectory. A PCA percentage of $45 \%$ was assigned to all mice that could not step on test day, since this is the lowest PCA score that we have ever encountered.

\section{EXPERIMENTAL RESULTS}

The average total number of steps performed in a best 15 second interval by the animals in each group is shown in Fig. 5. The "window" and "band" groups performed more steps than the fixed group. However, there does not seem to be any statistical difference between the two AAN training groups.

In Fig. 6, the inverse of the FWHM is plotted to emphasize the improvement in step rhythm throughout the study. The "window" and "band" AAN training groups consistently stepped more rhythmically than the fixed training group. Although the two variable groups could not be statistically distinguished, the "window" group seemed to recover

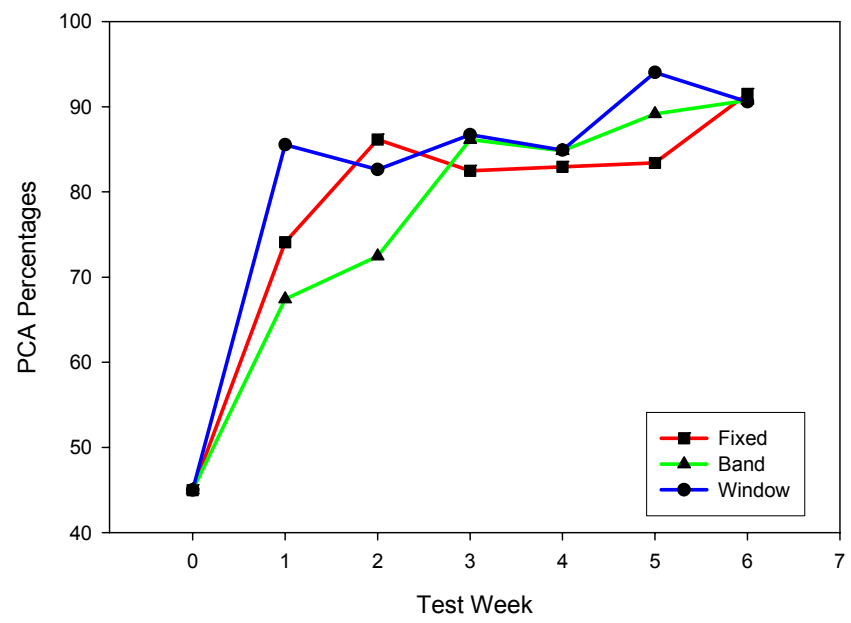

Figure 7: The PCA scores through out training faster. A score of zero was assigned to all mice that could not step on the first test day.

The PCA percentage score, which measures the spatial regularity of stepping, is plotted in Fig. 7. Although the "window" training group appeared to recover faster, there was no indication of a difference between the step shape consistencies of the three groups. This may be due to the small number of subjects. Alternatively, this supports previous findings that quipazine, rather than the specific training pattern, is primarily responsible for shape consistency [13]. Note that training of any form, combined with quipazine, substantially improves step regularity.

\section{DISCUSSION}

A key objective of this study was to ascertain whether providing variability in training enhances stepping recovery. A significant concern is that fixed trajectory training strategies may drive the spinal cord into a state of "learned helplessness," $[6,20]$ whereby the spinal cord, not challenged to explore potential stepping patterns on its own, will defer to the trainer and give up on learning. No statistically significant differences were observed between the variable "band" and "window" training groups. This may be due to the small $n$ used. Nevertheless, both variable training paradigms appeared to be a more effective rehabilitative strategy then the fixed training paradigm.

Nevertheless, interlimb coordination must be controlled in order to maximize locomotor recovery [21]. We found a similar result is true for adult spinal mice. Although mice trained with the "band" pattern recovered greatly in their stepping performance, their steps were typically arrhythmic and frequently interrupted by dragging. In contrast, stepping executed by the "fixed" and "window" trained groups were rhythmic and prolonged. This result is clearly seen in the FFT data, which show that stepping in the latter two groups converged to a frequency near $1 \mathrm{~Hz}$ as the study progressed, a result consistent with constant speed treadmill locomotion at $3 \mathrm{~cm} / \mathrm{sec}$.

Principal components analysis did not elucidate a difference in the shape consistency of the three groups. By week 3 , the average PCA score of all three groups was greater than $80 \%$, indicating that all of the mice could step rhythmically. This result supports previous observation that quipazine rather than step training has the larger influence on step shape consistency when the effect of quipazine alone was examined [13, 22].

Sensory information is critical to motor learning [22, 23]. The pattern and timing of assistance provided during step training dictates the extent to which the injured spinal cord can recover motor function. The results suggests that failure to enforce contralateral phasing of the legs during training limits stepping recovery compared to mice trained with an alternating gait. It is likely that the spinal cord is similarly highly sensitive to other proprioceptive cues. As demonstrated here, poorly conceived or poorly implemented training algorithms may actually inhibit stepping recovery. 
This emphasizes a fundamental advantage of using robotic devices that can consistently and accurately apply defined locomotor training paradigms while simultaneously maintaining a thorough quantitative record of both training and performance parameters. Used with data optimization schemes, quantitative data are teaching us which parameters to focus on when devising novel training strategies. By developing our understanding of how sensory inputs affect spinal learning, we can "tune the spinal cord to learn."

\section{Acknowledgements:}

This work is supported by the Christopher Reeve Paralysis Foundation, the National Institutes of Child Health and Humane Development and the National Institute of Neural Disorders and Stroke.

\section{REFERENCES}

[1] S. Hesse, H. Schmidt, C. Werner, and A. Bardeleben, "Upper and lower extremity robotic devices for rehabilitation and for studying motor control," Curr Opin Neurol, vol. 16, pp. 705-10, 2003.

[2] R. D. de Leon, M. D. Kubasak, P. E. Phelps, W. K. Timoszyk, D. J. Reinkensmeyer, R. R. Roy, and V. R. Edgerton, "Using robotics to teach the spinal cord to walk," Brain Res Brain Res Rev, vol. 40, pp. 267-73, 2002.

[3] D. J. Reinkensmeyer, J. L. Emken, and S. C. Cramer, "Robotics, motor learning, and neurologic recovery," Аnnu Rev Biomed Eng, vol. 6, pp. 497525, 2004.

[4] S. E. Fasoli, H. I. Krebs, and N. Hogan, "Robotic technology and stroke rehabilitation: translating research into practice," Top Stroke Rehabil, vol. 11, pp. 11-9, 2004.

[5] N. F. Skinner, "Learned helplessness: performance as a function of task significance," $J$ Psychol, vol. 102, pp. 77-82, 1979.

[6] R. N. Wool, D. Siegel, and P. R. Fine, "Task performance in spinal cord injury: effect of helplessness training," Arch Phys Med Rehabil, vol. 61, pp. 321-5, 1980.

[7] M. Wirz, R. Hornby, R. Rupp, and V. Dietz, "Locomotor training with a driven gait orthosis in incomplete spinal cord injury," Gait Posture, vol. Volume 21, pp. S74, 2005.

[8] "Spinal Cord Injury Information Network," [online], Available: $\quad$ http://www.spinalcord.uab.edu/ show.asp?durki $=21446,2005$, (Accessed 20 September 2005).

[9] V. R. Edgerton, R. D. Leon, S. J. Harkema, J. A. Hodgson, N. London, D. J. Reinkensmeyer, R. R. Roy, R. J. Talmadge, N. J. Tillakaratne, W. Timoszyk, and A. Tobin, "Retraining the injured spinal cord," J Physiol, vol. 533, pp. 15-22, 2001.

[10] N. J. Tillakaratne, M. Mouria, N. B. Ziv, R. R. Roy, V. R. Edgerton, and A. J. Tobin, "Increased expres- sion of glutamate decarboxylase $(\operatorname{GAD}(67))$ in feline lumbar spinal cord after complete thoracic spinal cord transection," J Neurosci Res, vol. 60, pp. 219-30, 2000.

[11] H. Barbeau and S. Rossignol, "Recovery of locomotion after chronic spinalization in the adult cat," Brain Res, vol. 412, pp. 84-95, 1987.

[12] R. D. de Leon, J. A. Hodgson, R. R. Roy, and V. R. Edgerton, "Full weight-bearing hindlimb standing following stand training in the adult spinal cat," $J$ Neurophysiol, vol. 80, pp. 83-91, 1998.

[13] A. J. Fong, V. R. Edgerton, L. L. Cai, C. K. Otoshi, W. K. Timoszyk, M. Merlo, A. J. Bigbee, H. Zhong, R. R. Roy, D. J. Reinkensmeyer, and J. W. Burdick, "Effects of quipazine and robotic training on spinal mice, pp.," Washington, DC: Soc Neurosci Abstr, vol. Program No. 498.20, 2003.

[14] V. R. Edgerton, N. J. Tillakaratne, A. J. Bigbee, R. D. de Leon, and R. R. Roy, "Plasticity of the spinal neural circuitry after injury," Annu Rev Neurosci, vol. 27, pp. 145-67, 2004.

[15] H. Barbeau and S. Rossignol, "Initiation and modulation of the locomotor pattern in the adult chronic spinal cat by noradrenergic, serotonergic and dopaminergic drugs," Brain Res, vol. 546, pp. 250-60, 1991.

[16] M. R. Brumley and S. R. Robinson, "The serotonergic agonists quipazine, CGS-12066A, and alphamethylserotonin alter motor activity and induce hindlimb stepping in the intact and spinal rat fetus," Behav Neurosci, vol. 119, pp. 821-33, 2005.

[17] E. S. Landry and P. A. Guertin, "Differential effects of 5-HT1 and 5-HT2 receptor agonists on hindlimb movements in paraplegic mice," Prog Neuropsychopharmacol Biol Psychiatry, vol. 28, pp. 1053-60, 2004.

[18] P. A. Guertin, "Role of NMDA receptor activation in serotonin agonist-induced air-stepping in paraplegic mice," Spinal Cord, vol. 42, pp. 185-90, 2004.

[19] D. Orsal, J. Y. Barthe, M. Antri, D. FeraboliLohnherr, A. Yakovleff, M. Gimenez y Ribotta, A. Privat, J. Provencher, and S. Rossignol, "Locomotor recovery in chronic spinal rat: long-term pharmacological treatment or transplantation of embryonic neurons?," Prog Brain Res, vol. 137, pp. 213-30, 2002.

[20] J. W. Grau, D. G. Barstow, and R. L. Joynes, "Instrumental learning within the spinal cord: I. Behavioral properties," Behav Neurosci, vol. 112, pp. 1366-86, 1998.

[21] S. F. Donker and P. J. Beek, "Interlimb coordination in prosthetic walking: effects of asymmetry and walking velocity," Acta Psychol (Amst), vol. 110, pp. 265-88, 2002.

[22] A. J. Fong, L. L. Cai, C. K. Otoshi, D. J. Reinkensmeyer, J. W. Burdick, R. R. Roy, and V. R. Edgerton, "Spinal cord-transected mice learn to step 
in response to quipazine treatment and robotic training," J Neurosci, vol. 25, pp. 11738-47, 2005.

[23] V. Dietz, R. Muller, and G. Colombo, "Locomotor activity in spinal man: significance of afferent input from joint and load receptors," Brain, vol. 125, pp. 2626-34, 2002.

[24] A. L. Behrman and S. J. Harkema, "Locomotor training after human spinal cord injury: a series of case studies," Phys Ther, vol. 80, pp. 688-700, 2000 .

\section{Appendix A: Computation of the Band Vector Fields}

To generate the velocity fields for the "Band" algorithm seen in Fig. 3, we transformed a simple convergent velocity field for a circular trajectory to the desired stepping trajectory via a numerical conformal mapping procedure.

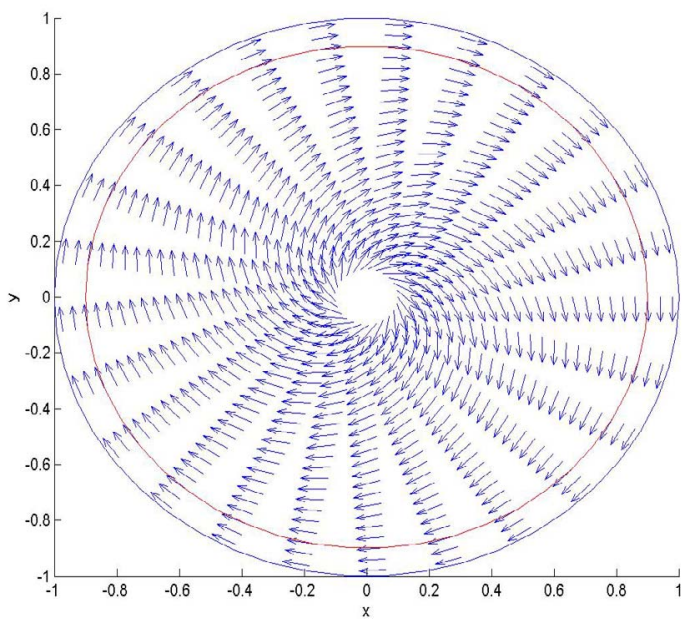

Figure 8: Convergent velocity field of a unit circle

Fig. 8 shows a clockwise outward spiraling velocity field inside a unit circle which will cause a point in the interior that follows the integral of the velocity fields to converge to the circular trajectory. The velocity field is determined by the following equations in polar coordinates $r, \alpha$ :

$$
\begin{aligned}
& \vec{v}_{r}(r, \alpha)=(1-\delta-r)(\vec{i} \cos (\alpha)+\vec{j} \sin (\alpha)), r \leq 1-\delta \\
& \vec{v}_{r}(r, \alpha)=0, \quad r>1-\delta \\
& \vec{v}_{a}(r, \alpha)=k(-\vec{i} \sin (\alpha)+\vec{j} \cos (\alpha))
\end{aligned}
$$

Distance $(0<\delta<1)$ defines a band along the unit circle. If the point is within the distance $\delta$ to the unit circle, the velocity field just follows the trajectory. Outside the band, the velocity field tends to move towards the band.

By reversing the direction of the angular component of the velocity field and mapping it onto the area outside the training trajectory, the inward spiraling velocity field component of Fig. 3 is realized.

The numerical conformal mapping is implemented via the Zipper program developed by Prof. D. E. Marshall of
University of Washington (http://www.math.washington.edu/ $\sim$ marshall/zipper.html). 